ARQGA / 1087

\title{
DIVERTÍCULO ÚNICO DO CECO: experiência de um hospital geral brasileiro*
}

\author{
Aljamir Duarte CHEDID ${ }^{1,2}$, Luciano Amaral DOMINGUES ${ }^{\mathbf{1}}$, Marcio Fernandes CHEDID ${ }^{\mathbf{3}}$, \\ Maitê de Mello VILLWOCK ${ }^{1}$ e Antônio Renato MONDELO ${ }^{1}$
}

RESUMO - Racional - A diverticulite cecal é uma condição rara, especialmente em populações ocidentais. Sua importância reside no fato de fazer parte do diagnóstico diferencial da apendicite aguda e do carcinoma ulcerado de ceco. Casuística e Métodos - Apresenta-se a experiência de um hospital geral do sul do Brasil no tratamento da diverticulite cecal. Descrevem-se quatro casos de divertículo único inflamado de ceco. Um destes teve diagnóstico pré-operatório através de tomografia computadorizada de abdome, tendo o paciente tratado clinicamente com remissão do quadro de diverticulite. Os outros três pacientes foram submetidos a colectomia direita com íleotransverso anastomose. Resultados - A mortalidade foi nula e nos casos operados não houve complicações pós-operatórias. Quando se consegue obter diagnóstico pré-operatório, pode-se optar por manejo clínico. Conclusão - Recomendamos laparotomia exploradora quando não há certeza diagnóstica. Preconizamos manejo cirúrgico radical quando o diagnóstico é efetuado através de laparotomia.

DESCRITORES - Doenças do ceco. Diverticulite, cirurgia. Colectomia.

\section{INTRODUÇÃO}

A diverticulite cecal é condição rara que se apresenta como um dilema diagnóstico para o cirurgião. Desde POTIER ${ }^{(16)}$, que descreveu o primeiro caso de diverticulite cecal, há alguns relatos, inclusive no $\mathrm{Brasil}^{(8)}$.

A prevalência de diverticulite cecal é baixa em populações ocidentais, sendo mais elevada nos descendentes de asiáticos. Na população ocidental, a maior parte dos divertículos cecais são solitários, segundo GRAHAM e BALLANTYNE(7).

Acredita-se que os divertículos solitários são congênitos, surgindo como uma projeção sacular do ceco na sexta semana de gestação ${ }^{(23)}$. Esses são considerados divertículos verdadeiros porque têm todas as camadas da parede colônica, incluindo a muscular. Estudos patológicos freqüentemente são inábeis para identificar a parede muscular do divertículo solitário devido à concomitância de alterações inflamatórias. Divertículos múltiplos são vistos mais comumente na população de origem asiática e são relatados como divertículos falsos, em sua grande parte ${ }^{(20)}$.
O divertículo solitário de ceco geralmente se localiza medialmente, próximo à válvula ileocecal e habitualmente não apresenta associação com diverticulose de outras porções do cólon ${ }^{(2,21)}$.

Pacientes com diverticulite cecal têm apresentação clínica praticamente indistinguível de apendicite aguda com febre, leucocitose e dor abdominal em quadrante inferior direito. A apresentação clínica da diverticulite cecal também pode ser a mesma do carcinoma ulcerado de ceco envolvendo o apêndice cecal. Desse modo, o correto diagnóstico préoperatório raramente é obtido ${ }^{(12)}$.

\section{CASUÍSTICA}

\section{Caso 1}

Paciente MSMB, 35 anos, feminino, branca, previamente hígida, foi admitida com o quadro de dor abdominal em quadrante inferior direito, de forte intensidade, iniciada 24

\footnotetext{
* Serviço de Cirurgia Geral do Hospital Ernesto Dornelles de Porto Alegre, RS.

Serviço de Cirurgia Geral do Hospital Ernesto Dornelles de Porto Alegre; ${ }^{2}$ Programa de Transplante Hepático Adulto do Hospital de Clínicas de Porto Alegre da ${ }^{3}$ Faculdade de Medicina da Universidade Federal do Rio Grande do Sul, Porto Alegre, RS.

Endereço para correspondência: Dr. Aljamir Duarte Chedid - Rua Marechal Andréa, 300/201 - Bairro Boa Vista - 91340-400 - Porto Alegre, RS. E-mail: aljamir@terra.com.br
} 
horas antes da internação. A dor era precipitada pela movimentação com aumento gradual de sua intensidade.

O exame físico revelou dor à palpação profunda e à descompressão súbita do quadrante inferior direito. Temperatura axilar de $38^{\circ} \mathrm{C}$, contagem de leucócitos de 14.000 x 106/L e 10\% de bastonados, hemoglobina de $12,8 \mathrm{~g} / \mathrm{dL}$ e hematócrito de $38 \%$. O exame qualitativo de urina (EQU) foi normal.

O diagnóstico presuntivo foi de apendicite aguda. Porém, durante a laparotomia, observou-se massa localizada no ceco próximo à válvula ileocecal, de aproximadamente $4 \mathrm{~cm}$ de diâmetro, que estava perfurada e tamponada pelo omento maior. Havia aumento do volume dos linfonodos do mesocólon. Devido à suspeita de malignidade, foi realizada hemicolectomia direita com íleo transverso anastomose primária términoterminal. A evolução pós-operatória foi sem intercorrências.

O exame anatomopatológico revelou divertículo verdadeiro único de ceco com diverticulite aguda necrosante e perfuração tamponada pelo omento maior, observando-se peritonite aguda focal e periapendicite aguda focal.

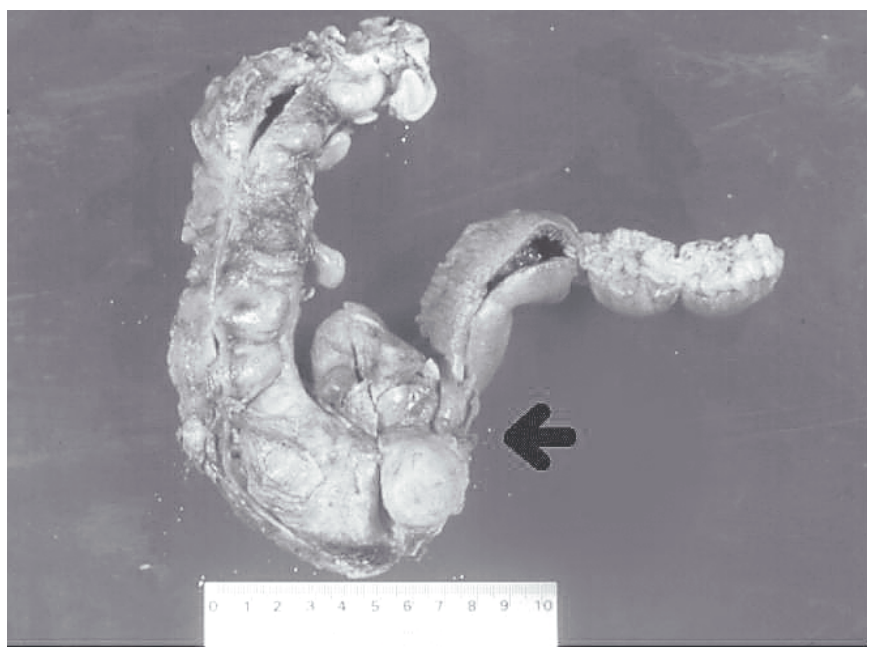

FIGURA 1 - Espécime de hemicolectomia direita demonstrando divertículo único de ceco (paciente do caso 1)

\section{Caso 2}

Paciente JDA, 47 anos, masculino, branco, admitido com quadro de dor abdominal em fossa ilíaca direita, iniciada há 3 dias, com piora progressiva de intensidade, associada à febre e a fezes diarréicas. Realizada apendicectomia há 15 anos.

Ao exame apresentava abdome distendido, timpanismo à percussão abdominal, defesa abdominal, dor à palpação abdominal profunda, principalmente em fossa ilíaca direita, sem dor à descompressão súbita. Temperatura axilar de $37,2^{\circ} \mathrm{C}$, hemoglobina de $13,4 \mathrm{~g} / \mathrm{dL}$, hematócrito de $38,8 \%$, contagem leucocitária com
$19.300 \times 106 / \mathrm{L}$ com $5 \%$ de bastonados. O EQU estava normal. Foi realizada ecografia abdominal, que evidenciou grande espessamento de paredes do ceco, saculação de sua parede lateral, com conteúdo ecogênico, medindo $2,5 \mathrm{~cm}$ x 1,6 cm, circundada por gordura omental infiltrada. Diagnóstico ecográfico: provável diverticulite cecal. A seguir, foi realizada tomografia abdominal, que revelou espessamento parietal e infiltração da gordura pericecal, que sofreu discreta impregnação pelo meio de contraste, de aspecto compatível com processo inflamatório cecal. Foi optado por tratamento conservador, iniciando-se antibioticoterapia com ampicilina, gentamicina e metronidazol, obtendo-se completa resolução do quadro em 6 dias.

\section{Caso 3}

Paciente OICV, 51 anos, feminino, branca, iniciou há 2 dias com quadro de dor abdominal periumbilical, que se localizou na fossa ilíaca direita, associada à anorexia. Não apresentava febre, náuseas, vômitos ou alterações do hábito intestinal.

Ao exame, apresentava abdome flácido, dor à palpação da fossa ilíaca direita, sem sinais de irritação peritonial. Apresentava temperatura axilar de $36,7^{\circ} \mathrm{C}$, hemoglobina de $13,4 \mathrm{~g} / \mathrm{dL}$, hematócrito de $39,6 \%$, contagem leucócitária $6.900 \times 106 / \mathrm{L}$ com 2\% (de neutrófilos bastonados). A ecografia abdominal demonstrou lesão arredondada e tubular de $2 \mathrm{~cm}$, com intensa sombra acústica, podendo estar relacionada a quadro de apendicite aguda. A seguir, foi realizada tomografia computadorizada abdominal, que revelou espessamento parietal do ceco e infiltração da gordura adjacente, podendo representar processo inflamatório, não sendo descartada doença neoplásica. Também se identificou imagem arredondada de $1,0 \mathrm{~cm}$, que pode estar relacionada com fecalito no interior da luz do apêndice cecal.

Foi indicada laparotomia exploradora durante a qual foi identificada massa de consistência endurecida de aproximadamente $3 \mathrm{~cm}$ de diâmetro no ceco. Devido à suspeita de malignidade foi realizada hemicolectomia direita com anastomose primária ileocólica términoterminal. A evolução pós-operatória foi sem intercorrências.

O exame anatomopatológico evidenciou divertículo único de ceco, com diverticulite crônica supurativa e periviscerite.

\section{Caso 4}

Paciente LSB, 26 anos, masculino, branco, previamente hígido, foi admitido com quadro de dor abdominal em fossa ilíaca direita, com evolução de 4 dias, de forte intensidade, com piora progressiva, associada à febre. Negava náuseas, vômitos e mudança de hábito intestinal.

$\mathrm{O}$ exame físico revelou dor à palpação profunda e à descompressão súbita da fossa ilíaca direita. Temperatura axilar de $36^{\circ} \mathrm{C}$, contagem de leucócitos de 13.400 x 106/L e 20\% de bastonados, hemoglobina de $14,5 \mathrm{~g} / \mathrm{dL}$, hematócrito de 43,8\%. A ecografia abdominal evidenciou 
imagem hipoecóica com 2,6 cm de calibre, arredondada, alongada, com intensa sombra acústica central, podendo estar relacionada à apendicite aguda.

Foi indicada laparotomia exploradora, durante a qual foi observada massa localizada no ceco, de aproximadamente $2,5 \mathrm{~cm}$ de diâmetro, ulcerada. Havia aumento do volume dos linfonodos do mesocólon. Devido à suspeita de malignidade, foi realizada hemicolectomia direita com íleo transverso anastomose primária términoterminal. A evolução pós-operatória foi sem intercorrências.

O diagnóstico anatomopatológico foi divertículo de ceco, com inflamação crônica abscedida, hemorragia e periviscerite. Linfadenite crônica em linfonodos retrocecais e mesocólicos.

\section{DISCUSSÃO}

A maioria dos pacientes com diverticulite de ceco se apresenta com dor abdominal indistinguível de apendicite aguda. O diagnóstico pré-operatório requer alto índice de suspeição, especialmente em pacientes jovens, com ancestrais asiáticos, que apresentam dor em quadrante inferior direito por mais de 24 horas de duração, sem náuseas, vômitos ou anorexia, e paciente apendicectomizado ${ }^{(1,4,9,13)}$.

A radiografia de abdome pode revelar fecalito em até 50\% dos casos e enema opaco pode ajudar na delimitação do divertículo com ou sem fecalito ${ }^{(22)}$. A dificuldade de visualizar o divertículo não exclui a possibilidade de sua presença, pois o lúmen diverticular pode ser obstruído por um fecalito ou por edema do colo do divertículo ${ }^{(6,19)}$. RAO et al. ${ }^{(17)}$ avaliaram a tomografia computadorizada para o diagnóstico diferencial de dor abdominal em quadrante inferior direito e encontraram sensibilidade de $98 \%$ e especificidade de $98 \%$ no diagnóstico de apendicite aguda. Além disso, demonstraram que a tomografia computadorizada seria um exame com boa relação custobenefício a ser empregado no diagnóstico diferencial dos quadros de dor abdominal com suspeita de apendicite aguda, o que se traduziu na redução de explorações cirúrgicas apendiculares negativas.

Quando a laparotomia revela apêndice normal e uma massa inflamatória no ceco, a suspeita clínica não deve ser direcionada somente para malignidade, mas também para diverticulite cecal. Outras condições no diagnóstico diferencial são doença de Crohn, actinomicose, perfuração por corpo estranho, amebíase, tumor carcinóide e tuberculose ${ }^{(18)}$

Algumas manobras intra-operatórias têm sido sugeridas para o correto diagnóstico e apropriado tratamento ${ }^{(3)}$. A mobilização do ceco pode auxiliar durante a investigação. Palpação direta da lesão pode revelar um fecalito, sugerindo diverticulite cecal, e palpação indireta através da parede intestinal oposta pode permitir a palpação do óstio diverticular ${ }^{(6,19)}$. Alguns autores ${ }^{(3,11)}$ recomendam cecotomia e biopsia de congelação; outros, porém, argumentam que este procedimento pode aumentar o risco de contaminação fecal e, em caso de malignidade, pode haver disseminação de células neoplásicas. Para divertículo solitário sem reação inflamatória apenas diverticulectomia simples com apendicectomia en passant, tem sido proposto ${ }^{(11,13,14,19)}$.

Se diagnóstico conclusivo de diverticulite cecal é obtido antes da realização de cirurgia, tratamento conservador com antibióticos de amplo espectro pode ser apropriado. Alguns autores recomendam que, se um divertículo cecal é reconhecido durante a cirurgia, ele deve ser deixado in situ e tratado somente com antibióticos de amplo espectro ${ }^{(5,6,10)}$, conforme a conduta adotada no caso 2 da presente casuística.

Estudo de revisão encontrou um correto diagnóstico intra-operatório em somente $65 \%$ dos pacientes submetidos a hemicolectomia direita de emergência ${ }^{(16)}$. Em outra revisão, esse índice foi de $84 \%{ }^{(15)}$. Nesta situação, diagnóstico de câncer não pode ser apropriadamente excluído. Adicionando-se a isso, se o divertículo é mantido, existe risco de recurrência de $15 \%$ a $71 \%{ }^{(7,9)}$. Finalmente, estudos retrospectivos têm mostrado que a hemicolectomia direita de emergência é segura para diverticulite cecal, com taxa de mortalidade de $1,4 \%{ }^{(7)}$, tendo sido realizada nos três pacientes em que não se obteve diagnóstico pré-operatório.

Recomenda-se laparotomia exploradora quando não há certeza diagnóstica pré-operatória, especialmente em pacientes idosos. Em virtude da dificuldade no diagnóstico transoperatório e de o diagnóstico diferencial envolver doença maligna, aconselha-se hemicolectomia direita para massas cecais inflamadas descobertas no transoperatório.

Chedid AD, Domingues LA, Chedid MF, Villwock MM, Mondelo AR. Solitary diverticulum of the cecum: experience of a Brazilian general hospital. Arq Gastroenterol 2003;40(4):216-219.

ABSTRACT - Background - Cecal diverticulitis is a rare condition, specially in western people. Its importance concerns of being part of the differential diagnosis of acute appendicitis and ulcerated cecal carcinoma. Aim - To present the experience of southern Brazilian general hospital in the treatment of cecal diverticulitis. Material and Methods - We present four cases of single inflamed cecal diverticulum. One was diagnosed by pre-operatively computer tomography and was treated medically without complications. The other three cases were diagnosed during operation and treated by right hemicolectomy and ileotransverse anastomosis. Results - There were no deaths or complications. When cecal diverticulitis is pre-operatively diagnosed it may be treated medically. We preclude laparotomy when the diagnosis is uncertain. Conclusion - We recommend radical surgical management when the diagnosis is made during operation.

HEADINGS - Cecal diseases. Diverticulitis, surgery. Colectomy. 


\section{REFERÊNCIAS BIBLIOGRÁFICAS}

1. Anscombe AR, Keddie NC, Schofield PF. Solitary ulcers and diverticulitis of the caecum. Br J Surg 1967;54:553-7.

2. Arrington, Tjudd S. Caecal diverticulitis. Am J Surg 1981;142:56-9.

3. Bell GA. Acute diverticulitis of the cecum. Am Surg 1974:40:370-2.

4. Burgess CM. Diverticulitis of the cecum. Am J Surg 1940;50:108.

5. Cutajar CL. Solitary cecal diverticula. Dis Colon Rectum 1978;21:627-9.

6. Fischer MG, Farkas AM. Diverticulitis of the cecum and ascending colon. Dis Colon Rectum 1984;27:454-8.

7. Graham SM, Ballantyne GH. Cecal diverticulitis. A review of the American experience. Dis Colon Rectum 1987;30:821-6.

8. Guimarães AS, Aprilli F, dos Santos Jr JC, Gomes DR, Carril CF, Barbieri-Neto J. Solitary inflammatory diverticulum of the cecum. Report of 8 cases. AMB Rev Assoc Med Bras 1981;27:346-8.

9. Harada RN. Surgical management of cecal diverticulitis. Am J Surg 1993;166:666-71.

10. Jorgensen SP. Caecal diverticulitis. Scand J Gastroenterol 1972;7:157-60

11. Laimon H. Diverticulitis of the cecum: a report of 8 cases. Am J Surg 1962;103:146-9.

12. Lane JS, Sarkar R, Schimit PJ, Chandler CF, Thompson JE Jr. Surgical approach to cecal diverticulitis. J Am Coll Surg 1999;188:629-34.
13. Lauridsen J. Acute diverticulitis of cecum. Arch Surg 1952;64:320.

14. Norfray JS. Cecal diverticulitis in young patients. Gastrointest Radiol 1980;5:37982.

15. Oudenhoven LF, Koumans RK. Right colonic diverticulitis: US and CT findings. Radiology 1998;208:611-8

16. Potier F. Diverticulite and appendicite. Bull Mem Soc Anat Paris 1912 37:29-31

17. Rao PM, Rhea JT. Effect of computed tomography of the appendix on treatment of patients and use of hospital resources. N Engl J Med 1998;338:141-6.

18. Rasmussen I. Acute solitary diverticulitis of the caecum. Acta Chir Scand 1988;154:399-401.

19. Schuler JG, Bayley J. Diverticulitis of the cecum. Surg Gynecol Obstet 1983;156:743-8.

20. Sugihara K, Muto T. Diverticular disease of the colon in Japan. A review of 615 cases. Dis Colon Rectum 1984;27:531-7.

21. Tan EC, Tung KH, Tan L, Wee A. Diverticulitis of caecum and ascending colon in Singapore. J R Coll Surg Edinb 1984;29:373-6.

22. Wagner D, Zollinger R. Diverticulitis of the cecum and ascending colon. Arch Surg 1961;83:124-31.

23. Waugh TR. Appendix vermiformis duplex. Arch Surg 1941;42:311-20.

Recebido em 6/12/2002. Aprovado em 20/3/2003 